\title{
Community Vital Signs: Taking the Pulse of the Community While Caring for Patients
}

\author{
Lauren S. Hughes, MD, MPH, MSc, Robert L. Phillips Jr., MD, MSPH, \\ Jennifer E. DeVoe, MD, DPhil, and Andrew W. Bazemore, MD, MPH
}

In 2014 both the Institute of Medicine and the National Quality Forum recommended the inclusion of social determinants of health data in electronic health records (EHRs). Both entities primarily focus on collecting socioeconomic and health behavior data directly from individual patients. The burden of reliably, accurately, and consistently collecting such information is substantial, and it may take several years before a primary care team has actionable data available in its EHR. A more reliable and less burdensome approach to integrating clinical and social determinant data exists and is technologically feasible now. Community vital signs_-aggregated community-level information about the neighborhoods in which our patients live, learn, work, and play_convey contextual social deprivation and associated chronic disease risks based on where patients live. Given widespread access to "big data" and geospatial technologies, community vital signs can be created by linking aggregated population health data with patient addresses in EHRs. These linked data, once imported into EHRs, are a readily available resource to help primary care practices understand the context in which their patients reside and achieve important health goals at the patient, population, and policy levels. (J Am Board Fam Med 2016; 29:419-422.)

Keywords: Population Characteristics, Public Health, Residence Characteristics, Social Determinants of Health

Physicians rarely enter an examination room without access to a patient's blood pressure, pulse, respiratory rate, and temperature-pieces of information so key to informing health decisions they are called "vital signs" (VSs). While patients' VSs provide a glimpse into their physical wellness, we lack analogous information about the neighborhoods in which they live, learn, work, and playpatients' community VSs. Community VSs, such as poverty level, education attained, or employment

This article was externally peer reviewed.

Submitted 22 May 2015; revised 24 February 2016; accepted 25 February 2016.

From the Pennsylvania Department of Health, Harrisburg (LSH); the American Board of Family Medicine, Lexington, KY (RLP); OCHIN, Inc., Portland, OR (JED); Department of Family Medicine, Oregon Health \& Science University, Portland (JED); and The Robert Graham Center, Washington, DC (AWB).

Funding: none.

Conflict of interest: none declared.

Corresponding author: Lauren S. Hughes, MD, MPH, MSc, Pennsylvania Department of Health, Health \& Welfare Building, 625 Forster St, 8th Floor West, Harrisburg, PA 17120 (E-mail: laurenshughes@gmail.com). status, could be independent social determinants of health, or they could be indices of these factors. ${ }^{1-4}$ Either way, community VSs convey contextual social deprivation and associated risks based on where patients live, and this is important information that could influence point-of-care decisions, similar to physiologic vital signs. We suggest that currently available information technologies using population-level data allow us to integrate these social elements into clinically useful community VSs without burdening providers.

Over the past several years, the volume and statistical sophistication of studies examining the relationship between neighborhood factors and individual health have increased. ${ }^{5-7}$ Clinicians and researchers have recognized that purely individual characteristics may not capture fully all determinants of health status. ${ }^{8}$ Policymakers and population health experts increasingly focus on initiatives that target nontraditional health care levers, such as housing, to mitigate chronic disease risk. ${ }^{8}$ Appreciating the neighborhood context renders a greater understanding of baseline vulnerabilities that our 
patients and populations face, risks and benefits of living in a particular place that accrue over time. This is not to say that neighborhood data should replace individual patient data or is inherently of more or less quality. Rather, these different types of data provide us with complementary, actionable information and perspectives. Frankly, there is a real need for research to understand the interplay of individual and neighborhood characteristics, and which of each are most important for understanding health outcomes. In the meantime, clinical judgment is the best means of interpolating individual and neighborhood factors, of which the latter could increase or decrease personal risk of chronic disease or injury over time but to different degrees.

Democratization of data and access to geospatial technologies and "big data" enable the creation of community VSs by using already available aggregated population health data at the zip code, census tract, or city block level and linking this information to geocoded patient address data within electronic health records (EHRs). Examples of population health data sources include vital statistics, disease surveillance, and census information. These linked data, once imported into EHRs, would help primary care practices understand the context in which their patients reside, increase awareness of resultant health risks, and tailor clinical or community interventions. Using current geocoding technologies to create a community VS geocoding application programming interface that can accept a data request for all addresses among an identified clinic patient population is one approach to integrating these data into patients' medical records. The application programming interface can then assign detailed geographic identifiers and the associated community VSs to each patient address. This information could be included in patient registries at the practice level, and/or it could be imported into the EHR and displayed in patient records at the point of care.

In 2014 both the Institute of Medicine (IOM) ${ }^{9}$ and the National Quality Forum ${ }^{10}$ recommended the inclusion of social determinants of health data in EHRs. The IOM and National Quality Forum reports primarily focus on collecting socioeconomic and health behavior data directly from individual patients. This approach to data collection will be tedious, lengthy, and potentially burdensome to individual practices. Without significant work to create standard and streamlined processes, the formatting of these patient-reported data would likely vary widely among practices, and the subsequent data may be incomplete, inconsistent, and unreliable. This important work needs to be done, but it will take time, and we should work on an immediate parallel and complementary approach that leverages preexisting, standardized sources of population health data to create community VSs now. An approach that administratively geocodes and directly integrates neighborhood data predictors of health into EHRs could be done relatively quickly, achieve administrative efficiencies, minimize provider burden, and enable uniform comparisons across practices. Furthermore, this community VS approach is a practical health information technology solution that fosters a stronger link between primary care and public health to improve patient and population well-being, fulfilling the IOM's 2012 charge to better integrate primary care and public health through greater collaboration, data sharing, and alignment between these 2 fields. ${ }^{11}$ Community VSs also offer a specific goal for the newest IOM Vital Signs report for creating point-of-care measures that can support clinical decisions. $^{12}$

Clinically, community VSs may influence discussions between physicians and patients about health goals, safety, and community-based resources. Knowing social risk may inform the clinical recommendations physicians offer. A prime example of this is the Healthe $R x$ project at the University of Chicago, a patient-centered community services e-prescribing initiative offered in Chicago's South Side neighborhoods. Funded by a grant from the Center for Medicare and Medicaid Innovation, this program connects patients with community resources they need to help them manage chronic diseases. ${ }^{13}$ After each clinic visit, patients receive a customized map of local health and social resources that is individually tailored to a patient's address and diagnoses and is generated by the EHR. All community resource recommendations are pulled from a large database known as Healthe Rx, which includes housing and transportation, mental health, fitness and nutrition, and substance abuse counseling services. Data for Healthe $R x$ are supplied largely by MAPSCorps, a summer internship for local high school and university students to conduct community asset mapping. Instead of simply recommending that patients 
eat better and exercise more, primary care clinicians are able to help patients connect to resources they can use on a regular basis. Healthe $R x$ has created stronger ties with community businesses and organizations that use the database to influence programming priorities.

Community VSs could also help primary care providers better understand variations in the health status of their local populations and determine where to prioritize their attention. Primary care practices could map "hot spots" of disease prevalence or high utilization of expensive services, as well as map "cold spots" where few health care services or supports exist. ${ }^{14,15}$ Knowing these community VSs can help primary care providers become more aware of and involved in targeted population health interventions. The Indiana Network for Patient Care (INPC) exemplifies this application well. INPC is one of the country's oldest and most comprehensive EHR systems, providing point-of-care data to more than 14,000 physicians, containing records dating back more than 30 years, and integrating up to 1 million clinical transactions daily from more than 200 sources. ${ }^{16}$ The 11 -county Indianapolis area also boasts the nation's largest community information system, known as Social Assets and Vulnerabilities Indicators (SAVI), which details more than 10,000 community-level characteristics over the past 35 years, including public safety, education, human services, housing, and demographic data. The joint INPC-SAVI team has now started geocoding all clinical records and tagging them with geospatial attributes that permit linkages to a diverse array of neighborhood-level data through SAVI. This merged data system serves several purposes, including the study of health inequities in the social determinants of sexually transmitted infections.

Building on the Indiana example, the American Board of Family Medicine (ABFM) aims to develop a similar capacity for its diplomates as part of Part IV of Maintenance of Certification. By creating tools that enable access to and use of community VSs, the ABFM plans to study whether these resources help family physicians make better decisions for their patient populations and improve outcomes. The ABFM considers critically important the creation of educational resources and opportunities for family medicine students, residents, and currently practicing physicians to better understand social determinants of health and how to apply this knowledge to improve population health. A secondary goal is to align Maintenance of Certification tools with likely requirements for capturing social determinant data.

In addition to helping advance patient and population health at an individual practice level, incorporation and use of community VSs can also help achieve community or regional health goals and contribute to large comparative effectiveness research studies. An example of these broader applications of community VSs can be found in the first widespread test of this concept currently being studied by OCHIN, a community health information network based in Portland, OR, and The Robert Graham Center, a primary care policy research group in Washington, DC. Using funding from the Patient-Centered Outcomes Research Institute, these partners are testing integration of community VS data into the EHR data warehouse of the ADVANCE (Accelerating Data Value Across a National Community Health Center Network) Clinical Data Research Network of PCORnet, a national Patient-Centered Outcomes Research Institute-funded network for conducting patientcentered outcomes research. ${ }^{17}$ The linkage of these data at the population level will enhance PCORnet's comparative effectiveness research efforts. In the future this data system could also support practicelevel access to the linked clinical and community VS data through EHRs, allowing providers and administrators a greater ability to target chronic disease interventions for both individual patients and the communities in which they live.

All data systems have their limitations, including the challenges of data sharing, privacy agreements, and the maintenance of data integrity, quality, and accuracy. Nevertheless, there are places nationwide where this data convergence is happening successfully. Health Insurance Portability and Accountability Act-compliant geocoding-and integration within EHRs—are both feasible and scalable, given proper vendor and provider incentives. As technological solutions become more facile and the use of community VSs more widespread, it will be essential to examine whether their application improves health outcomes and lowers chronic disease risk within our communities. It will also be important to evaluate how community VSs shape policy development, direct community resource allocation, spur clinic-community collaboration, and inform 
value-based payment approaches with respect to social determinants of health.

We have long known that place matters to personal and population health. Neighborhood-level social determinants of health have begun to shape local public health and policy interventions. It is now time for we as family physicians to harness the power of community VSs to improve the health of our patients and our communities.

\section{References}

1. Butler D, Petterson S, Phillips R, Bazemore A. Measures of social deprivation that predict health care access and need within a rational area of primary care service delivery. Health Serv Res 2013;48:539-59.

2. Salmond C, Crampton P, King P, Waldegrave C. NZiDep: a New Zealand index of socioeconomic deprivation for individuals. Soc Sci Med 2006;62: 1474-85.

3. Welsh Government. Welsh Index of Multiple Deprivation (WIMD). December 1, 2015. Available from: http://gov.wales/statistics-and-research/welshindex-multiple-deprivation/?lang=en. Accessed February 20, 2016.

4. Department for Communities and Local Government. English indices of deprivation. December 13, 2012, updated September 30, 2015. Available from: https://www.gov.uk/government/collections/englishindices-of-deprivation\#documents). Accessed February 20, 2016.

5. Crampton P, Foley J. Why are we weighting? Equity considerations in primary health care resource allocation formulas. In: Dew K, Metheson A, eds. Understanding Health Inequalities in Aotearoa, New Zealand. Dunedin: Otago University Press; 2008:133-145.

6. Commission to Build a Healthier America. Where we live matters for our health: neighborhoods and health. Issue brief 3: Neighborhoods and health. New Brunswick, NJ: Robert Wood Johnson Foundation; 2008. Available from: http://www.commissiononhealth. org/PDF/888f4a18-eb90-45be-a2f8-159e84a55a4c/
Issue \% 20Brief\% $203 \% 20$ Sept $\% 2008 \% 20$ - \% 20 Neighborhoods\%20and\%20Health.pdf. Accessed February 24, 2016.

7. US Department of Housing and Urban Development. Understanding neighborhood effects of concentrated poverty. Evidence Matters. Winter 2011. Available from: https://www.huduser.gov/portal/ periodicals/em/winter11/highlight2.html. Accessed February 24, 2016.

8. Roux A, Mair C. Neighborhoods and health. Ann N Y Acad Sci 2010;1186:125-45.

9. Institute of Medicine. Capturing social and behavioral domains in electronic health records: phase 1 . Washington, DC: The National Academies Press; 2014.

10. National Quality Forum. Multi-stakeholder input on a national priority: improving population health by working with communities - Action guide 1.0. Washington, DC: National Quality Forum; 2014.

11. Institute of Medicine. Primary care and public health: exploring integration to improve population health. Washington, DC: The National Academies Press; 2012.

12. Institute of Medicine. Vital signs: core metrics for health and health care progress. Washington, DC: The National Academies Press; 2015.

13. HealtheRx.org [homepage on the Internet]. Accessed January 22, 2015.

14. Gawande A. The hot spotters. New Yorker, January 24, 2011, 40-51.

15. Westfall JM. Cold-spotting: linking primary care and public health to create communities of solution. J Am Board Fam Med 2013;26:239-40.

16. Comer K, Grannis S, Dixon B, et al. Incorporating geospatial capacity within clinical data systems to address social determinants of health. Public Health Rep 2011;126(Suppl 3):54-61.

17. DeVoe JE, Gold R, Cottrell E, et al. The ADVANCE network: accelerating data value across a national community health center network. J Am Med Inform Assoc 2014;21:591-5. 